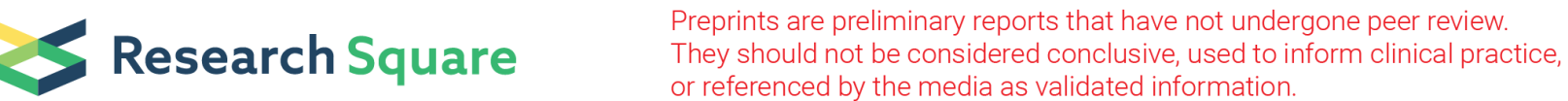

\section{Trends In Frailty And Its Associated Factors In Community Dwelling Elderly Indian Population During COVID-19 Pandemic- A Prospective Analytical Study.}

\section{Karan Gautam}

Kasturba Medical college, Mangalore

Shyam Krishnan ( $\sim$ krish.shyam@manipal.edu )

Kasturba Medical college, Mangalore

Vijaya Kumar

Kasturba Medical college, Mangalore

Megha Nayak

Kasturba Medical college, Mangalore

\section{Research Article}

Keywords: Frailty, COVID-19 pandemic, Sarcopenia, Functional Mobility

Posted Date: June 21st, 2021

DOl: https://doi.org/10.21203/rs.3.rs-605962/v1

License: (c) (i) This work is licensed under a Creative Commons Attribution 4.0 International License.

Read Full License

Version of Record: A version of this preprint was published at F1000Research on March 14th, 2022. See the published version at https://doi.org/10.12688/f1000research.70638.1. 


\section{Abstract}

\section{Background}

There is a scarcity of quality literature on the prevalence of frailty among community dwelling elderly in India. This study was originally planned to analyze the longitudinal trends in frailty status of community dwelling elderly in an Indian population as well to identify factors associated with frailty in the Indian context. However the recruitment phase of this study coincided with one of the largest lockdowns in history, associated with the COVID-19 Pandemic, and this gave us a unique opportunity to study the effects this pandemic enforced, absolutely necessary restrictions, had on the frailty status as well the factors affecting frailty in elderly.

\section{Methods}

A prospective observational study was designed and conducted amongst 19 community dwelling elderly of Dakshina Kannada District, in Karnataka India. Outcome variables of frailty(EPIF), Physical activity(PASE), functional mobility(TUG), gait speed(10 meter walk test), nutritional status(MNAT), body composition(BIA), and strength(dynamometry), were measured at baseline and on follow-up exactly after 3 months. The change occurring in these variables over the 3 month period was analyzed and the change in frailty was independently correlated with changes in each of the other outcomes.

\section{Results}

We couldn't identify any statistically significant difference in frailty over a period of 3 months. However there was a highly significant change in the physical activity status, lower extremity muscle strength, body composition, functional mobility, gait sped, and cognitive function in the same time period.

\section{Conclusion}

Though individual determinants of frailty in community dwelling changed over a 3 month period, these changes failed to produce any observable/measurable difference in frailty status.

\section{Introduction}

Frailty derived from Latin word 'Fradilita' meaning Brittleness, is an important and emerging term in Geriatric medicine.(1) There is no definition which is internationally recognized to be valid, but it is usually associated with adverse outcomes developed as a consequence of increased venerability because of decline in physiological systems with increasing age, triggered by any minor stressor which collectively leads to sudden changes in state of health.(2)Frailty is geriatric syndrome which is multidimensional in nature. World Health Organization (WHO) defined frailty in 2017 as "a clinically recognizable state in which the ability of older people to cope with everyday or acute stressors is compromised by an increased vulnerability brought by age-associated declines in physiological reserve and function across multiple organ systems".(3) 
900 million people around the world comes under elderly age group according to WHO, out of which in India there are 104 million elderly (> 60 years age). It is also estimated that India will hold largest geriatric population around the globe by the year 2050.(4) With the advancement in medical sciences there is decrease in mortality rates, the life expectancy is increased and so is the frailty among the elderly.(5) It is estimated that $4-10 \%$ of elderly population dwelling in United state are frail, also $8.1 \%$ elderly are observed frail in United kingdom, 6.5\% and $7 \%$ in Italy and France (respectively).(6)

A large and compressive study by WHO showed that among middle and low income countries (South Africa, China, Russia, Ghana, India and Mexico) India has the highest prevalence of frailty (i.e. 56.9\%), more number of women are frail then men ( $47 \%$ of elderly men and $67 \%$ of elderly women). $(7,8)$

The evaluation of frailty is difficult because of lack of any standardized tool. There exists 67 tools for quantifying Frailty, out of which only 9 of the screening tools are highly cited (more than 200 citations). (9)Phenotype of frailty (given by Fried)(10) and Frailty index (given by Mintnitski)(11) are the validated and most widely used screening tools.(9) Fried Phenotype of frailty model defines a person frail when 3 or more than 3 physiological deficits out of 5 are present (10), whereas Frailty index model expresses frailty as a "ratio of existing deficits to the total probable deficits there could be". Wide ranges of diseases, disabilities, signs and symptoms are defined as these deficits.(11)

Ageing leads to numerous changes in physiological systems of our body which are fundamental to the growth of frailty, specifically the immunological system, the neuromuscular system and neuroendocrine system.(12)These changes in body interact progressively and adversely, leading to loss of physiological function and reserve (state of compromised homeostasis).(12) The risk factors for frailty are varied and have been found to have multiple linear and non linear interactions. For example consequence of normal ageing leads to loss of muscle mass and strength.(13) Muscle loss can also be accelerated due to chronic illness, poor nutrition, decrease in growth hormone production, and reduced physical activities. (13) All these factors are inter-related to each other through complex interactions and ultimately leads to frailty. Socio economic and demographic variables like availability of disposable income/finances, level of education, nutritional status, and general living conditions have been found to be confounders of frailty.(7)

Several studies have identified factors like sarcopenia, loss of muscle strength, functional mobility and gait velocity changes, loss of weight, reduced physical activity and easy exhaustibility to be strong independent confounders of frailty. The most closely associated biological parameters of frailty have been identified, as inflammatory markers, dyslipidemic markers, endocrinological markers, insulin resistance and state of glycemia.(12)

Available literature on the feasibility of predicting frailty state that changes in functional as well as biological parameters could be used as well qualified candidates to estimate and quantify frailty. Out of these risk factors, it is purported that functionality could be the strongest predictor or measurer of frailty. Sarcopenia, connective tissue remodeling, and inflammatory markers mediated physiological and 
functional changes and their interaction among themselves and other confounders of frailty need to be studied to develop a predictive model of frailty.(14)

A recent large scale review on the state of frailty related research in India had stated that there is a rather alarming lack of conceptualization or epidemiological data regarding frailty in Indian scenario, and there is a dire need to identify the key confounders for frailty syndrome among Indian elderly.(7)There is a scarcity of quality literature on the prevalence of frailty among community dwelling elderly in India. This study was originally planned to analyze the longitudinal trends in frailty status of community dwelling elderly in an Indian population as well to identify factors associated with frailty in the Indian context. However the recruitment phase of this study coincided with one of the largest lockdowns in history, mandated to minimize the spread of COVID 19 in India. Restrictions were put in place to minimize the outdoor movement of population in general and specifically the elderly, who were recognized to be the most vulnerable group with respect to the pandemic. This gave us a unique opportunity to study the effects this pandemic enforced, absolutely necessary restrictions, had on the frailty status as well the factors affecting frailty in elderly.

\section{Methodology}

After obtaining necessary permission to recruit subjects, the study recruitment was planned to commence in March 2020. A total of 28 samples were screened between first and third week of March of whom 22 subjects were found to be eligible for study. The criteria for inclusion were that the age must be greater than 65 years, MOCA score greater than 26 at the time of first evaluation. Subjects with known diagnosis of any progressive disorder, as well as those with, cardiovascular, musculoskeletal, neurological or systemic illness which could potentially interfere with data collection were excluded.

Subjects' demographics as well as medical history were recorded using appropriate tools following which evaluation for frailty index questionnaire was administered to identify and quantify frailty among them. Lower extremity muscle strength was evaluated using baseline hand held dynamometer. A Tanita ${ }^{\circledR}$ Segmental Body Composition analyzer was used to determine the body composition variables of muscle mass, visceral fat and total body fat percentage for each subject. Subjects were then made to do a $10 \mathrm{~m}$ walk test to analyze the gait velocity following which the nutritional status and socioeconomic status were evaluated using MNAT-NESTLE $®$ and BG prasad scale respectively. Physical activity level was recorded using PASE scale following which a timed up and go test was then performed to analyze the functional mobility status following the collection of outcome measures each subject was given a date exactly 3 month from date of 1 st evaluation for the follow up assessment.

Unexpectedly India went into complete lockdown in the third week of March 2020 following the rise of COVID-19 cases which in its strict form lasted for approximately 70 days following which there was a phase wise gradual unlock. Our follow up data collection coincided with phase two of unlock but still there was a general advisory for elderly subjects to be home bound to minimize chances of exposure. Of 
the 22 subjects recruited only 19 subjects returned for the timely follow up evaluation. All the outcomes were again collected using same tool and in the same order at the end of third month.

\section{Data Analysis}

The data was collected and analyzed using JAMOVI version 1.6.14. Statistical software. Normality of continuous variables was tested using Shapiro-Wilk test. Demographic variables were expressed in terms of descriptive statistics. Differences in Gait velocity, strength, body composition and functional mobility at baseline and at 3 months of follow up was analyzed using Wilcoxon sign rank test/Students pared sample T-test. Changed scores of independent variables were individually associated with changes scores in "Evaluation of Frailty index for physical activity questionnaire" using Spearman's Correlation test.

\section{Results}

A total of 28 subjects were screened out of which 19 samples after fulfilling the inclusion and exclusion criteria were recruited. The demographic data of all participants are represented in table 1:

\begin{tabular}{|lll|}
\hline & & Mean \pm SD \\
\hline Age (years) & $74.2 \pm 8.13$ \\
\hline Height $(\mathrm{cm})$ & & $160 \pm 7.16$ \\
\hline Weight $(\mathrm{kg})$ & Baseline & $64.2 \pm 8.69$ \\
\cline { 2 - 3 } & 3 month follow-up & $64.3 \pm 8.62$ \\
\hline
\end{tabular}

Characteristics of patients such as Gender, Marital status, Height, level education is presented in table 2: 


\begin{tabular}{|c|c|c|}
\hline & & Percentage (n) \\
\hline \multirow[t]{2}{*}{ Gender } & Male & $63.2 \%(12)$ \\
\hline & Female & $36.8 \%(7)$ \\
\hline \multirow[t]{3}{*}{ Marital status } & Married & $57.9 \%(11)$ \\
\hline & Unmarried & $5.3 \%(1)$ \\
\hline & Widowed & $36.8 \%(7)$ \\
\hline \multirow[t]{6}{*}{ Education } & 1 (un-educated) & $0 \%(0)$ \\
\hline & 2 (1-5th standard) & $26.3 \%(5)$ \\
\hline & 3 (6-10th standard) & $31.6 \%(6)$ \\
\hline & 4 (11-12th standard) & $15.8 \%(3)$ \\
\hline & 5 (Graduate) & $26.3 \%(5)$ \\
\hline & 6 (Post-graduate) & $0 \%(0)$ \\
\hline \multirow[t]{5}{*}{ B G Prasad } & I (Rs.7008/Month and above) & $0 \%(0)$ \\
\hline & II (Rs.3504-7007/Month) & $0 \%(0)$ \\
\hline & III (Rs.2102-3503/Month) & $0 \%(0)$ \\
\hline & IV (Rs.1051-2101/Month) & $15.8 \%(1)$ \\
\hline & V (Rs.1050/Month and below) & $84.2 \%(18)$ \\
\hline
\end{tabular}

Change in outcome variables at baseline and at 3 month of follow up Table 3: 


\begin{tabular}{|lll|}
\hline VARIABLES & MEAN DIFFERENCE & p-VALUE \\
\hline Water Content & -0.0632 & 0.786 \\
\hline MOCA & 0.7368 & $0.007^{*}$ \\
\hline TUG & -0.64 & $0.001^{*}$ \\
\hline Body fat & -0.3632 & 0.009 \\
Visceral fat & -0.4211 & $0.004^{*}$ \\
\hline 10 m walk test & -1.097 & $0.029^{*}$ \\
\hline EPIF & 0.625 & 0.138 \\
\hline BMR & -3 & 0.815 \\
\hline Muscle mass & -0.55 & $0.00{ }^{*}$ \\
\hline PASE & 43 & $0.003^{*}$ \\
\hline *- statically significant & \\
\hline
\end{tabular}

Table 4

Difference between baseline muscle strength and 3 month follow up:

\begin{tabular}{|c|c|c|c|c|}
\hline \multirow[t]{2}{*}{ MUSCLE GROUP } & \multicolumn{2}{|l|}{ RIGHT } & \multicolumn{2}{|l|}{ LEFT } \\
\hline & Mean difference & p-value & Mean difference & p-value \\
\hline Shoulder flexion & 0.421 & 0.016 & 0.316 & $0.055^{\star}$ \\
\hline Shoulder Extension & -0.786 & 0.001 & 0.786 & $0.001 *$ \\
\hline Shoulder Adduction & 0.474 & 0.046 & 0.579 & $0.03 *$ \\
\hline Shoulder Abduction & -0.421 & 0.072 & -0.842 & $0.001 *$ \\
\hline Elbow flexion & -0.632 & $0.001^{*}$ & -0.632 & $0.001 *$ \\
\hline Elbow extension & 1.211 & $0.014^{*}$ & 1.158 & 0.287 \\
\hline Hip flexion & -0.263 & $0.001 *$ & -0.947 & $0.001 *$ \\
\hline Hip extension & 0.789 & $0.001 *$ & 1.053 & $0.001 *$ \\
\hline Hip Abduction & -1.053 & $0.001 *$ & -1 & $0.001 *$ \\
\hline Hip Adduction & 0.737 & $0.001 *$ & 0.895 & $0.001 *$ \\
\hline Knee flexion & -0.684 & $0.012^{*}$ & -1 & $0.001 *$ \\
\hline Knee Extension & 0.579 & $0.001 *$ & 0.632 & $0.001 *$ \\
\hline
\end{tabular}


Table 5

represents correlation between EPIF and outcome variables:

\begin{tabular}{|c|c|c|}
\hline VARIABLES & CORRELATION COEFFICIENT & p-value \\
\hline Weight & 0.119 & 0.628 \\
\hline MOCA & -0.044 & 0.859 \\
\hline MNATN & -0.143 & 0.560 \\
\hline TUG & -0.030 & 0.904 \\
\hline $10 \mathrm{M}$ Walk Test & 0.020 & 0.935 \\
\hline Body Fat & -0.239 & 0.297 \\
\hline Water Content & 0.235 & 0.333 \\
\hline Muscle Mass & 0.183 & 0.454 \\
\hline BMR & 0.171 & 0.483 \\
\hline Visceral Fat & 0.049 & 0.841 \\
\hline R Shoulder Flexion & 0.018 & 0.941 \\
\hline R Shoulder Extension & -0.274 & 0.255 \\
\hline R Shoulder Adduction & -0.203 & 0.404 \\
\hline R Shoulder Abduction & -0.114 & 0.642 \\
\hline L Shoulder Flexion & 0.053 & 0.828 \\
\hline L Shoulder Extension & 0.018 & 0.943 \\
\hline L Shoulder Adduction & -0.071 & 0.773 \\
\hline L Shoulder Abduction & 0.049 & 0.841 \\
\hline R Elbow Flexion & 0.114 & 0.644 \\
\hline R Elbow Extension & -0.717 & 0.443 \\
\hline L Elbow Flexion & 0.470 & 0.042 \\
\hline L Elbow Extension & 0.470 & 0.042 \\
\hline R Hip Flexion & -0.030 & 0.923 \\
\hline R Hip Extension & -0.219 & 0.368 \\
\hline R Hip Abduction & -0.029 & 0.907 \\
\hline R Hip Adduction & 0.135 & 0.582 \\
\hline L Hip Flexion & -0.152 & 0.533 \\
\hline
\end{tabular}




\begin{tabular}{|lll|}
\hline VARIABLES & CORRELATION COEFFICIENT & p-value \\
\hline L Hip Extension & 0.148 & 0.545 \\
\hline L Hip Adduction & -0.015 & 0.592 \\
\hline L Hip Abduction & -0.130 & 0.596 \\
\hline L Knee Flexion & -0.052 & 0.831 \\
\hline L Knee Extension & 0.220 & 0.366 \\
\hline R Knee Flex & 0.093 & 0.706 \\
\hline R Knee Ext & -0.257 & 0.289 \\
\hline
\end{tabular}

\section{Discussion}

Present study was undertaken primarily to analyze the trends in frailty status of a cohort of community dwelling elderly, residing in the Dakshina Kannada district of Karnataka state in India over a period of 3 months as well as to analyze the strength of association between change in frailty score and cognition, nutritional status, gait velocity, functional mobility, body mass, and strength The recruitment period of the study coincided with the beginning of ongoing SARS COVID 19 which proved to be a major hindrance in approaching, screening and evaluating elderly subjects. Over the period of study, a total of 28 subjects were screened, of which 22 fulfilled the criteria of inclusion in the study. However of the 22 subjects, the follow up evaluation could be done only for a total of 19 subjects and hence the goals of the study were realigned to investigate the influence of pandemic induced lockdown and the associated reduction in physical activity on the outcome variables. In the current study we found that there is an observable change in frailty status over a period of 3 months but it was not statically significant.

Frailty is an umbrella term and there are many tools to measure Frailty. EPIF scale was used in the current study because it covers all domains of frailty (like physical, psychological, social functioning and general health), and has been proven to have good reliability and validity.(15) The data collection involved administering 4 questionnaires (EPIF, MOCA, MNAT NESTLE $®$ ) which on an average took $45 \mathrm{~min}$ - 1 hour to complete. Objective measures of strength, functional mobility, gait velocity, and body composition analysis would take an additional hour to complete. This made the entire data collection process a time consuming one thereby adversely affecting the number of subjects recruited in a day. However other than the afore mentioned 3 subjects who chose to forgo the follow-up evaluation because of the pandemic situation, there were no additional dropouts in the span of study and no reported discomfort or adverse event pertaining to data collection.

The primary objective of the study was to detect any association between changes in frailty status and other outcome variables. It must be noted that there was only very minimal difference (over a period of 3 months) in Frailty score (mean difference 0.625 ) and findings were not statically significant. We could 
not find any statically significant relationship between changes in frailty score and the changes in strength, muscle mass, cognition, nutritional status, gait velocity, or functional mobility.

However it must be emphasized that, when the independent variables were compared at baseline and 3 month of follow-up there was a statistically significant difference found in the scores of MOCA, TUG, visceral fat, PASE and muscle mass. The muscle mass and gait velocity showed a marginal but statically significant reduction, whereas total body fat as well as visceral fat content showed an increment. Cognitive functions as measured by MOCA and gait velocity (implied by an increase in time taken to complete $10 \mathrm{M}$ walk test) showed a decline in the above mentioned period, whereas the time taken to complete TUG had marginally increased. The observed differences in MOCA scores though were never sufficient to imply a cognitive decline. It can be inferred from these finding that a short span of 3 months has brought about measurable differences in variables which have been previously associated with frailty.

Previous research corroborated our findings in that there is a definitive decline in muscle mass ranging between 2 to $4 \%$ annually in older men and women of all ethnicity. There is also a concurrent increase in body fat content averaging about $0.8 \%$ within the same time span.(16)

Factors that influence body composition, especially muscle mass include genetic variables, metabolic variables, endocrinological variables, co-morbidities, diet, alcoholism, smoking, as well as gender and ethnicity. It must be emphasized however that physical activity as an independent variable is a strong predictor for loss of muscle mass and changes in body composition in elderly.(17)The data collection of present study coincided with the period of pandemic enforced restriction and all of the recruited subjects had reported a considerable decline in the amount of physical activity they indulged in the same period. For measuring physical activity we had used Physical activity Scale for elderly (PASE) and we found a highly significant reduction in the physical activity (Mean Difference $=43, p<0.05$ ) over the period of 3 months. For the population which we had studied, the major source of physical activity used to be walking in public places like parks or attending organized social gatherings like yoga and group exercise sessions. Since most of these activities were deemed to be unsafe, especially in elderly population, there was virtually a complete absence of these activities in the lockdown period.

Our data analysis shows there is a statically significant decline in functional mobility as measured by TUG with ageing, but it must be emphasized that this decline was barely consequential and for all intentions and purpose it is safe to assume there was no decline in functional mobility of the studied cohort. Gait velocity showed a statically significant but minuscule difference when compared over a period of 3 months.

In all major muscle groups of lower extremity, there was a significant difference noted in strength which ranged from a difference of $0.7 \mathrm{~kg}$ to $1.5 \mathrm{~kg}$. One of the key associated finding was that the decline in strength of bilateral hip and knee musculature (hip abductors, hip adductors, knee extension of right side and knee extensors, hip flexors, hip extensors and hip adductors on the left side) showed statically significant moderate correlation with decline in muscle mass. Previous studies have shown that there is 
insufficient evidence of a liner relationship between the loss of muscle strength and muscle mass in aging, though both have been individually established as definitive outcomes of ageing. Other factors affecting muscle strength have been identified as impaired reciprocal inhibition, alteration in rate coding of motor unit activation, as well as changes in metabolic characteristic of muscle fibers. These changes can happen independent of the changes in muscle mass.(18)The changes in muscle strength could then be attributed to the definitive decline in physical activity levels as previously stated, which would have precipitated a deconditioning/reversal effect on muscle strength. In our study cohort we observed neither statically significant nor any amount of change in the nutritional status of study population as measured by MNAT®.

\section{Conclusion}

Two key findings of this study are that, 1) there has been a definitive decline in physical activity of elderly within the lockdown period, and, 2) there is absolutely no significant change in the frailty status of community dwelling elderly, even in a time period characterized by physical activity restrictions due to the COVID 19 induced lockdown, though some of the independent determinants of frailty showed a decline in the same period. Present study failed to establish any association between frailty and changes in cognitive, functional mobility, body composition, strength, or nutritional factors, during a relatively short span of 3 months.

\section{Abbreviations}

WHO- World health organization

GS - Gait speed

SAGE - Study on global ageing and adult health

MOCA - Montreal cognitive assessment

TUG - Timed up and go

MNA - Mini nutritional assessment

\section{Declarations}

\section{Ethics approval and consent to participate}

The study was approved by the Scientific and Institutional Ethics committee of KMC Mangalore (IEC KMC MLR 11-19/590). All stages of the study were conducted in strict adherence to the principles of "Helsinki Declaration" for research on human subjects. All subjects were explained the objectives of the study in a language which they fully comprehended, and were recruited for the study only after signing a written informed consent. 


\section{Consent for publication}

Not Applicable.

\section{Availability of data and materials}

The datasets used and/or analyzed during the current study are available from the corresponding author on reasonable request.

\section{Competing interests}

The authors declare that they have no competing interests.

\section{Funding}

The authors hereby declare that we have received no external funding at any stage of this research. All aspects of this study has been funded by the researchers themselves.

\section{Authors' contributions}

SK, VK and KG were involved in conceptualization of the study.

$\mathrm{KG}$, and $\mathrm{MN}$ did the data collection and Data compilation.

SK did the data analysis.

KG, SK, VK and MN collaborated in the manuscript preparation.

I hereby declare that all Authors have thoroughly read and approved the Manuscript.

\section{Acknowledgements}

Not Applicable

\section{References}

1. Walston J, Hadley EC, Ferrucci $L$ et al. Research agenda for frailty in older adults: toward a better understanding of physiology and etiology: summary from the American Geriatrics Society/National Institute on Aging Research Conference on Frailty in Older Adults. J Am GeriatrSoc 2006;54:9911001.

2. Clegg A, Young J. The Frailty Syndrome. Clinical Medicine 2011;11:72-5.

3. World Health Organisation (2017): WHO Clinical Consortium on Healthy Ageing. Report of consortium meeting 1-2 December 2016 in Geneva, Switzerland. Geneva.

4. World Health Organisation (2015): World Health Statistics 2015. 
5. Frailty syndrome: implications and challenges for health care policy - UCL Discovery.

6. Nguyen T, Cumming R, Hilmer S. A Review of Frailty in Developing Countries. The journal of nutrition, health \&amp; aging 2015;19:941-946.

7. Das S. Frailty syndrome: a problem lurking in indian geriatric population.

8. Biritwum RB, Minicuci N, Yawson AE et al. Prevalence of and factors associated with frailty and disability in older adults from China, Ghana, India, Mexico, Russia and South Africa. Maturitas 2016;91:8-18.

9. Frailty assessment instruments: Systematic characterization of the uses and contexts of highly-cited instruments. - Abstract - Europe PMC.

10. Fried LP, Tangen CM, Walston J et al. Frailty in older adults: evidence for a phenotype. J Gerontol A BiolSci Med Sci 2001;56:M146-156.

11. Mitnitski AB, Mogilner AJ, Rockwood K. Accumulation of deficits as a proxy measure of aging. ScientificWorldJournal 2001;1:323-36.

12. Fried L, Walston J.(2003). Frailty and failure to thrive. In: Hazzard W, Blass J, Halter J, et al., editors. Principles of geriatric medicine and gerontology. 5th edn. New York: McGraw-Hill. 11(1): 72-75

13. Roubenoff R. Sarcopenia: a major modifiable cause of frailty in the elderly. J Nutr Health Aging 2000;4:140-2.

14. Fulop T, Larbi A, Witkowski JM et al. Aging, frailty and age-related diseases. Biogerontology 2010;11:547-63.

15. Salbach NM, Jaglal SB, Williams Jl. Reliability and validity of the evidence-based practice confidence (EPIC) scale. J ContinEduc Health Prof 2013;33:33-40.

16. Goodpaster BH, Park SW, Harris TB et al. The loss of skeletal muscle strength, mass, and quality in older adults: the health, aging and body composition study. J Gerontol A BiolSci Med Sci 2006;61:1059-64.

17. Curtis E, Litwic A, Cooper C et al. Determinants of Muscle and Bone Aging. J Cell Physiol 2015;230:2618-25.

18. Lynch GS. Sarcopenia - Age-Related Muscle Wasting and Weakness: Mechanisms and Treatments. Springer Science \& Business Media, 2010. 